\title{
Competitive Anterograde and Retrograde Atrioventricular Junctional Activation in Atrial Fibrillation
}

\author{
FRED H.M. WITTKAMPF** M.Sc., MIKE J.L. DE JONGSTE,** M.D., \\ and FRITS L. MEIJLER, $\$$ M.D.
}

From the *Heart-Lung Institute, Section of Cardiology, University Hospital of Utrecht, the **Department of Cardiology, Thoraxcenter, University Hospital of Groningen, and ${ }^{\S}$ The Interuniversity Cardiology Institute of the Netherlands, Utrecht, The Netherlands

\begin{abstract}
Reset and Cancellation. In eight medically-treated patients with chronic atrial fibrillation and a random ventricular rhythm, we studied the effect of single right ventricular stimuli delivered after each eighth spontaneous $R$ wave during at least 1 hour. The coupling interval of the extrastimulus was fixed and differed marginally from the shortest spontaneous RR interval. The histograms of spontaneous RR intervals and of the "compensatory" pauses following the ventricular extrasystoles were calculated. Analysis of these histograms and simulation of the interaction between anterograde and retrograde impulses in a computer model suggests that in seven of the eight patients the compensatory pause may be caused by two distinctly different mechanisms: (1) reset of the timing cycle of atrioventricular nodal activation by relatively early retrograde impulses; and (2) interception of anterograde impulses by relatively late ventricular extrasystoles. The finding that early retrograde impulses are not blocked by concealed atrioventricular nodal conduction makes the existence of decremental conduction and extinction of atrial impulses at different levels within the node unlikely. The results of this study support the hypothesis that the distal side of a weakly coupled junctional area inside the AV node behaves as a pacemaker for the ventricular rhythm during atrial fibrillation. ( $J$ Cardiovasc Electrophysiol, Vol. 1, pp. 448-456, October 1990)
\end{abstract}

atrial fibrillation, concealed conduction, compensatory pause

\section{Introduction}

The effect of blocked atrial impulses on subsequent atrioventricular (AV) conduction has been described in detail by Langendorf (1948). However, the precise mechanism of concealed (anterograde and retrograde) conduction is still not clear. On one hand, these phenomena have been explained by gradually changing properties of the AV node (Langendorf, 1948; Moe, et al., 1964; Langendorf, et al., 1965; Moore, 1967; Lehmann, et al., 1984). Others have hypothesized

This study was supported by the Wijnand M. Pon Foundation, Leusden, The Netherlands.

Address for correspondence: Fred H.M. Wittkampf, M.Sc., Heart-Lung Institute, Section of Cardiology, University Hospital Utrecht, 100 Heidelberglaan, 3584 CX, Utrecht, The Netherlands.

Manuscript received 13 April 1990; Accepted for publication 2 July 1990. that discontinuous conduction across a single localized region within the AV node is responsible for what is generally called concealed conduction (Rosenbleuth, 1958; Antzelevitch and Moe, 1983; Jalife, 1983; Billette, 1987; McKinnie, et al., 1989).

In a previous study, we made it plausible that during atrial fibrillation (AF), the AV node may act as an electrotonically modulated pacemaker (Wittkampf, et al., 1988). This hypothesis was seriously challenged by Dreifus and Mazgalev (1988).

Subsequently, making use of the compensatory pause in AF as a model to study concealed AV conduction, we found further evidence in support of our previous hypothesis (Meijler and Fisch, 1989; Wittkampf, et al., 1990). However, we only studied patients with $\mathrm{AF}$ and a relatively slow ventricular response. Despite the random- 
ness of their ventricular rhythm, one could not exclude that these patients may have had some sort of AV nodal abnormality, so that the effect of retrograde activation on the $\mathrm{AV}$ node might have been altered. We therefore repeated our observation in patients with "normal" or high ventricular rates. In these patients, retrograde penetration into the AV node is only possible if delivery of the extrastimulus fortuitously falls within an interval between anterograde impulses that would otherwise have been long. However, the occurrence of these longer intervals cannot be predicted during AF (Bootsma, et al., 1970; Brody, 1970). Premature ventricular stimulation, outside the refractory period of the His-Purkinje system in patients with AF and a fast ventricular rhythm, will therefore result in a mixed pattern of $\mathrm{AV}$ nodal penetration by the ventricular extrasystoles and collision with anterogradely conducted impulses. With computer simulation techniques as an investigational tool, the underlying mechanisms can be analyzed.

\section{Methods}

\section{Patient Study}

\section{Stimulation protocol}

To limit patient discomfort during this lengthy investigation, the study was only performed in patients with chronic AF and an already implanted multiprogrammable ventricular pacemaker. During spontaneous rhythm, single right ventricular extrastimuli were delivered, with a fixed coupling interval to the preceding $\mathrm{R}$ wave, by means of the implanted pacemaker using previously described techniques (Wittkampf, et al., 1990). The coupling interval of the extrastimulus was at least $50 \mathrm{msec}$ longer than the effective ventricular refractory period. This situation was maintained for 60 to 90 minutes during recording of the electrocardiogram on $\mathrm{fm}$ magnetic tape.

The following protocol was used: (1) Programming of the implanted pacemaker to the synchronous mode and a basic rate of $30 \mathrm{ppm}$ and 15-minute rest; (2) Ten-minute recording until approximately 500 consecutive spontaneous RR intervals were obtained; and (3) Sixty- to 90-minute recording with delivery of single right ventricular extrastimuli with a fixed coupling interval after each eighth $\mathrm{R}$ wave.

\section{Patient selection}

The criteria for patient selection were as follows: chronic AF and a random ventricular rhythm; and an implanted ventricular pacemaker that could be programmed to the synchronous mode and a basic rate of $30 \mathrm{ppm}$.

After informed consent, eight patients volunteered to be included in the study (Table 1). The histogram and autocorrelogram of all these patients confirmed a random ventricular rhythm. Indication for pacemaker implantation had been either sick sinus syndrome or AF with dizzy spells, syncope, or both. The patients with sick sinus syndrome all developed permanent AF after pacemaker implantation. All patients had some form of medical treatment that was maintained throughout the study.

TABLE 1

Relevant Clinical Patient Data

\begin{tabular}{|c|c|c|c|c|}
\hline $\begin{array}{l}\text { Patient } \\
\text { Number }\end{array}$ & $\begin{array}{c}\text { Age and } \\
\text { Sex }\end{array}$ & Symptoms* & $\begin{array}{c}\text { Antiarrhythmic } \\
\text { Agents }\end{array}$ & $\begin{array}{l}\text { Associated } \\
\text { Conditions }\end{array}$ \\
\hline 1 & $82 \mathrm{~F}$ & Syncope & Verapamil & SSS* \\
\hline 2 & $66 \mathrm{M}$ & DS & $\begin{array}{l}\text { Verapamil } \\
\text { Propanalol }\end{array}$ & - \\
\hline 3 & $68 \mathrm{M}$ & Syncope & Disopyramide & MVR \\
\hline 4 & $68 \mathrm{M}$ & & $\begin{array}{l}\text { Metoprolol } \\
\text { Digoxine }\end{array}$ & - \\
\hline 5 & $71 \mathrm{~F}$ & DS & $\begin{array}{l}\text { Metoprolol } \\
\text { Digoxine }\end{array}$ & SSS* \\
\hline 6 & $77 \mathrm{M}$ & & Digoxine & SSS* \\
\hline 7 & $75 \mathrm{M}$ & DS & $\begin{array}{l}\text { Digoxine } \\
\text { Propranolol }\end{array}$ & - \\
\hline 8 & $76 \mathrm{M}$ & DS & Digoxine & SSS* \\
\hline
\end{tabular}

$\mathrm{AF}=$ atrial fibrillation; $\mathrm{DS}=$ dizzy spells; $\mathrm{F}=$ female; $\mathrm{M}=$ male; $\mathrm{MVR}=$ mitral valve replacement; $\mathrm{SSS}=$ sick sinus syndrome. $*$ Symptoms before pacemaker implantation. 


\section{Analysis of the electrocardiogram}

The electrocardiogram was analyzed as described before (Wittkampf, et al., 1990). The coupling interval of the extrastimulus (RS) was defined as the delay between previous QRS onset and pacemaker extrastimulus, and SR as the interval between that stimulus and the onset of the next spontaneous QRS complex. The RR and SR intervals were measured, and the histograms of those intervals were calculated and plotted for each patient.

\section{Computer Simulation Study}

For our hypothesis to be correct, reset of the discharge sequence of the AV node will only occur if delivery of the extrastimulus fortuitously falls within an interval between anterograde impulses that would otherwise have been long. Other retrograde impulses will simply collide with and eliminate the anterograde impulses. The histogram of pauses following single premature ventricular complexes will thus reflect the results of a mixture of AV nodal resetting and anterograde impulse interception.

\section{Description of the model}

Assuming that the conduction velocity through the peripheral His-Purkinje system is constant, the ventricular rhythm (histogram of $R R$ intervals) during AF reflects the rhythm of the AV nodal discharge sequence. For each patient, the AV node can be simulated by a randomly irregular generator ("focus") with an interval histogram identical to that of the spontaneous RR intervals of that patient. Retrograde penetration of this "focus" resets its inherent timing cycle and thereafter a new random cycle length is calculated.

Another part of the model is a bidirectional delay that simulates the delay in the AV junction and beyond. The traveling time of anterograde and retrograde impulses can be set independently. Collision causes extinction of both impulses and does not affect the timing cycle of the AV nodal "focus."

The third and final part of the model represents the right ventricle ("ventricle"). The arrival of an anterograde impulse generates a spontaneous " $R$ wave."

The computer model also allows for the delivery of "ventricular" stimuli. In a first series of observations, single extrastimuli were de- livered with a predetermined coupling interval after every eighth spontaneous " $R$ wave." In a second series, stimulation is modified into an "on-demand" mode, with a programmable pacing interval. All ventricular intervals are measured and stored in the computer for further analysis.

A schematic drawing of the computer model is shown in Figure 1. A Lewis diagram of its behavior during the delivery of single extrastimuli is shown in Figure 2. During uninterrupted anterograde conduction, depolarizations of the AV nodal "focus" reach the "ventricle" after a constant anterograde delay. The "ventricular" rhythm is then identical to that of the "focus" (Fig. 2A). When the extrastimuli occur relatively shortly before the next expected spontaneous $\mathrm{R}$ wave, then collision and extinction of impulse wave fronts will follow (Fig. 2B). However, when the extrastimulus accidentally falls relatively early in the random timing cycle, then the "focus" will be reset by retrograde activation (Fig. 2C). This model thus enables analysis of the interaction between multiple randomly irregular anterograde and single premature retrograde impulses and measurement of the histogram of simulated postextrasystolic (SR) intervals. During on-demand pacing, "ventricular" stimuli are delivered whenever the interval between spontaneous $\mathrm{R}$ waves becomes longer than that of the programmed pacing interval.

\section{Simulation Model}

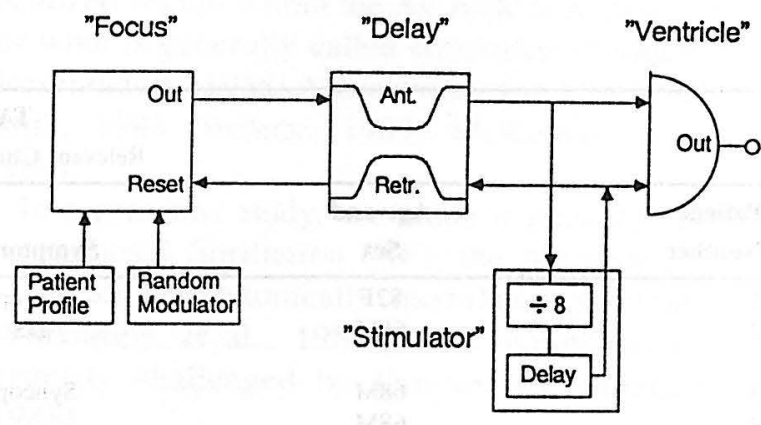

Figure 1. Schematic drawing of the simulation model. The "focus" generates a randomly irregular rhythm that is equal to that of a particular patient. Collision of anterograde and retrograde impulses within the "Delay" causes cancellation of both. Impulses at the output of the "Ventricle" are recorded for later analysis. "Ventricular" Stimuli can be delivered by the "Stimulator." 


\section{Lewis Diagram of Simulation Model}
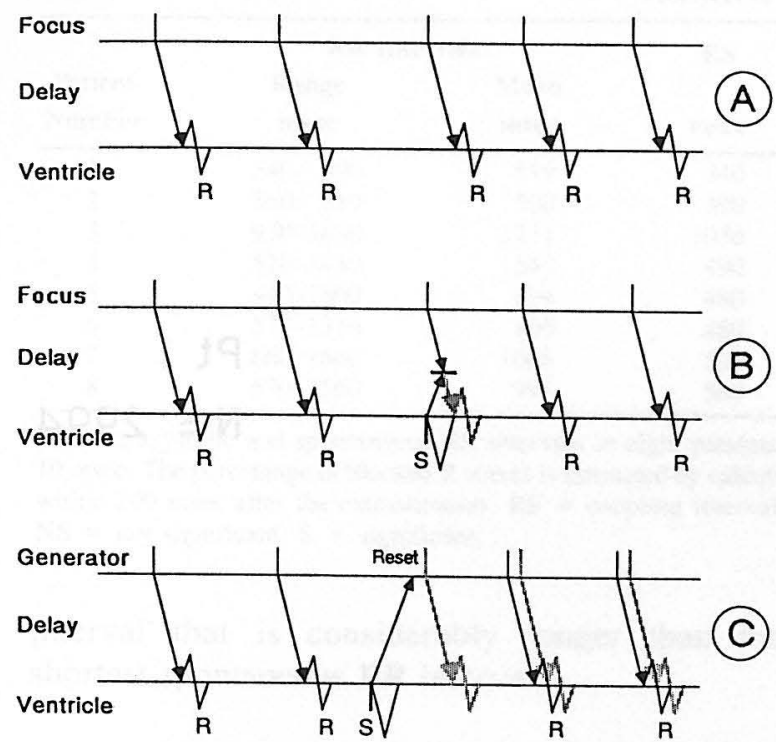

Figure 2. Lewis diagram of the performance of the simulation model. (A) Spontaneous anterograde conduction; (B) collision of an anterograde with a retrograde impulse; and $(\mathrm{C})$ reset of the generator timing cycle are shown.

For each patient, the histogram of spontaneous RR intervals is used in the "focus" to generate a random rhythm with an identical interval histogram. The coupling interval of the extrastimulus is set identical to the value used during the patient study protocol. For each patient, seven simulations were performed with different retrograde conduction delays between 0 and 300 msec in steps of $50 \mathrm{msec}$, whereas the anterograde conduction delay remained constant at $100 \mathrm{msec}$. The obtained histograms of SR intervals were compared with the truly measured histogram of SR intervals.

We have previously shown that in patients with chronic AF, right ventricular pacing is able to eliminate short RR intervals and to regularize the ventricular rhythm (Wittkampf, et al., 1988). Therefore, we performed a second series of simulation experiments to test the behavior of the model during on-demand "ventricular" stimulation. Again, the histogram of spontaneous RR intervals was used to generate the random rhythm. The stimulation protocol was similar to that of the original study and involved a stepwise decreasing pacing interval. At each step, 500 "ventricular" intervals were measured. During these simulations the retrograde conduction delay was arbitrarily set at $100 \mathrm{msec}$. All simulations were performed on a Macintosh SE computer (Apple Computer Inc., Cupertino, CA).

\section{Statistical analysis}

Comparison of histograms of true and simulated "compensatory" pauses was performed as described before (Wittkampf, et al., 1990). In short, the relative position of the two histograms was corrected for their mean interval duration. Thereafter, Kolmogorov-Smirnov analysis (Zar, 1974) was performed in an approach to reject the supposition that the two histograms are different. A P value of 0.05 was used as the level of statistical significance.

\section{Results}

\section{Patient Study}

The compensatory pause after single premature extrastimuli

The histograms of spontaneous RR intervals and of the postextrasystolic SR intervals of patient 1 are shown in Figures 3 and 4, respectively. In this case, the coupling interval of the extrastimulus is equal to the shortest spontaneous RR interval, and therefore a number of retrograde impulses will almost certainly have collided with anterograde impulses. Indeed, Kolmogorov-Smirnov analysis of the two histograms reveals that the difference in shape is statistically significant. The results of all eight patients are summarized in Table 2. In five of the eight patients, the shape of the histogram of SR intervals differed significantly from that of the spontaneous RR intervals.

\section{Computer Simulation Study}

The result of the simulation of patient 1 are shown in Figures 5 and 6. When no extrastimuli are delivered, the timing sequence of the simulated "spontaneous RR intervals" is random, and the histogram is equal to that of the actually measured spontaneous RR intervals (compare Figs. 3 and 5). The histogram of simulated SR intervals is shown in Figure 6. During this simulation, the anterograde delay was set at 100 msec, the retrograde conduction delay at 150 


\section{HISTOGRAM OF RR INTERVALS}

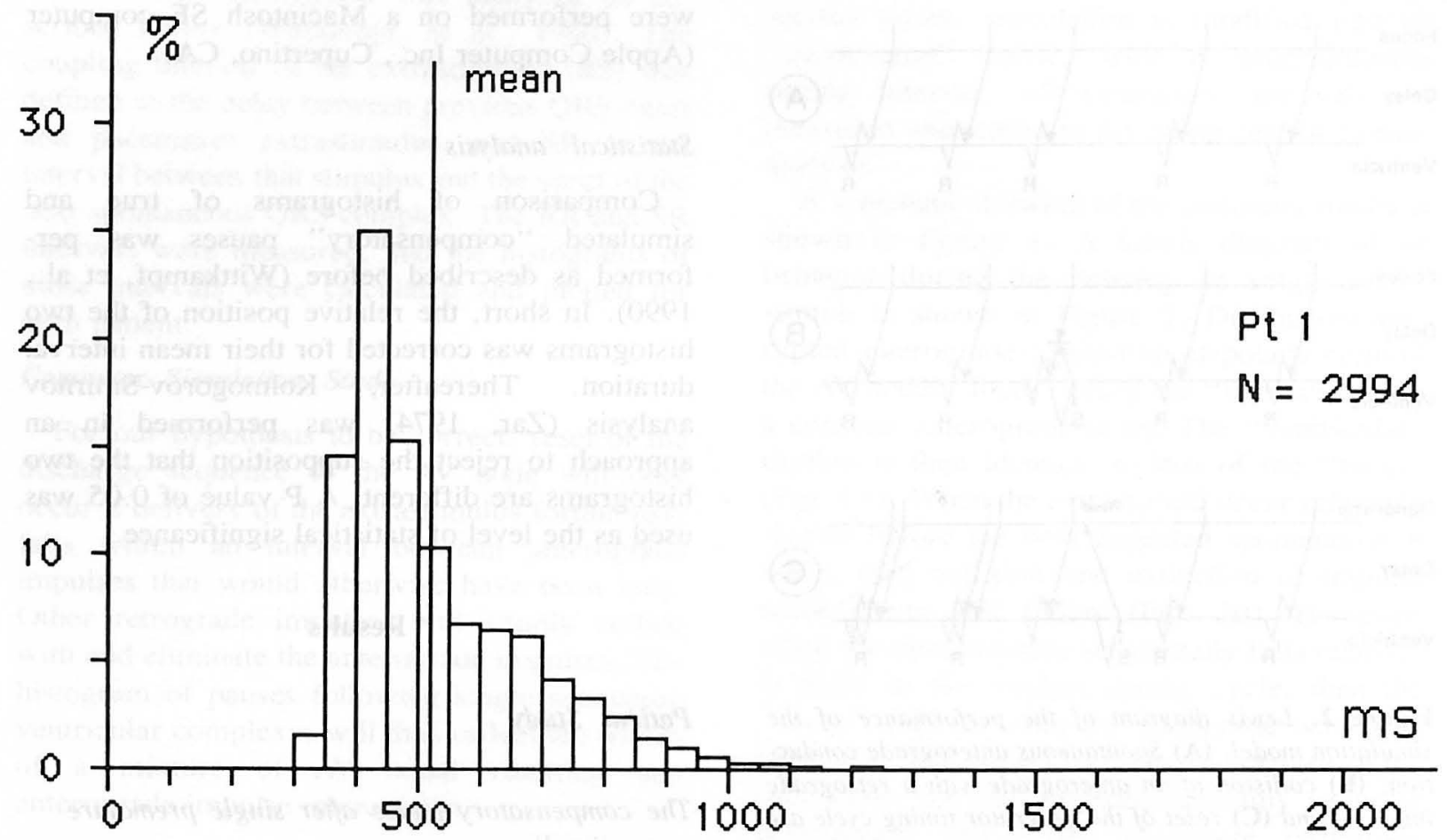

Figure 3. Histogram of almost 3,000 spontaneous RR intervals in pt 1. The intervals are measured during the period in which single extrastimuli were delivered after each eighth spontaneous $R$ wave.

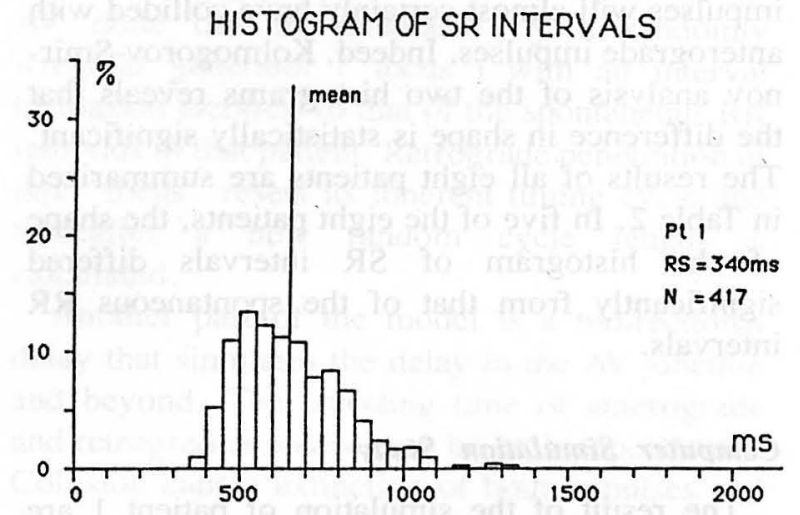

Figure 4. Histogram of SR intervals in pt 1 . The shape of the histogram is different from that of the histogram of spontaneous $R R$ intervals as shown in Figure 3. $R S=$ coupling interval between the onset of a spontaneous $R$ wave and the extrastimulus delivered by the implanted pacemaker; $S R=$ interval between extrastimulus from the implanted pacemaker until the onset of the next spontaneous $R$ wave. msec, and the coupling interval of the extrastimulus ("RS") at $340 \mathrm{msec}$, which is identical to the applied RS interval in this patient. Kolmogorov-Smirnov analysis of the two waveforms reveals that the difference in shape between both histograms is not statistically significant. The results of the simulations of all patients are summarized in Table 3 . In seven of the eight patients, we obtained histograms of simulated SR intervals that were not significantly different from those of the clinically obtained SR intervals. The range of values for the retrograde conduction delay that resulted in similarity of histogram shape is listed in Table 3 . The optimal value of retrograde delay, which we defined as a nonsignificant difference in histogram shape and a minimal difference between the mean measured and simulated SR interval, varied from 100 to 250 msec.

The behavior of the model during simulated "on-demand" ventricular stimulation is shown in Figure 7. A stepwise- decreasing pacing interval reduces the number of short $R R$ intervals until all spontaneous $\mathrm{R}$ waves are abolished at a pacing 
TABLE 2

Analysis of Ventricular Intervals

\begin{tabular}{|c|c|c|c|c|c|c|c|}
\hline \multirow[b]{2}{*}{$\begin{array}{l}\text { Patient } \\
\text { Number }\end{array}$} & \multicolumn{2}{|c|}{ RR Intervals } & \multirow{2}{*}{$\begin{array}{c}\text { RS } \\
\text { msec }\end{array}$} & \multicolumn{2}{|c|}{ SR Intervals } & \multirow{2}{*}{$\begin{array}{c}\text { Blocked } \\
\text { R waves } \\
\%\end{array}$} & \multirow{2}{*}{$\begin{array}{c}\text { Histogram } \\
\text { Shape } \\
\text { Difference }\end{array}$} \\
\hline & $\begin{array}{c}\text { Range } \\
\text { msec }\end{array}$ & $\begin{array}{c}\text { Mean } \\
\text { msec }\end{array}$ & & $\begin{array}{l}\text { Range } \\
\text { msec }\end{array}$ & $\begin{array}{c}\text { Mean } \\
\text { msec }\end{array}$ & & \\
\hline 1 & $340-890$ & 519 & 340 & $420-1020$ & 650 & 66 & $S$ \\
\hline 2 & $360-810$ & 500 & 390 & $350-910$ & 578 & 77 & $\mathrm{~S}$ \\
\hline 3 & $970-1630$ & 1251 & 1050 & $1040-1810$ & 1407 & 53 & $\mathrm{~S}$ \\
\hline 4 & $520-1430$ & 849 & 490 & $700-1650$ & 1067 & 20 & NS \\
\hline 5 & $440-1800$ & 894 & 480 & $460-1940$ & 1065 & 32 & $\mathrm{~S}$ \\
\hline 6 & $570-1310$ & 880 & 480 & $770-1510$ & 1126 & 17 & NS \\
\hline 7 & $660-1660$ & 1066 & 500 & $860-1810$ & 1282 & 5 & NS \\
\hline 8 & $670-1560$ & 993 & 500 & $840-1740$ & 1241 & 3 & S \\
\hline
\end{tabular}

Post-extrasystolic and spontaneous RR intervals in eight patients with atrial fibrillation. All timings except mean intervals are rounded to $10 \mathrm{msec}$. The percentage of blocked $\mathrm{R}$ waves is estimated by calculating the percentage of spontaneous RR intervals which would have occurred within $200 \mathrm{msec}$ after the extrastimulus. RS = coupling interval of the extrastimulus; $\mathrm{SR}=$ compensatory pause after the extrastimulus; NS = not significant; $\mathrm{S}=$ significant .

interval that is considerably longer than the shortest spontaneous RR interval.

\section{Discussion}

\section{Behavior of the simulation model}

In this study, we used a simplified model of impulse transmission through the AV junction. It was only made to investigate whether simple collisions of anterograde and retrograde impulses could account for the changes in the shape of the histogram of compensatory pauses relative to that of spontaneous RR intervals. We did not intend to simulate the filtering process of the fibrillatory atrial impulses itself. In the model, only the sum of the anterograde and retrograde conduction

\section{HISTOGRAM OF SIMULATED RR INTERVALS}

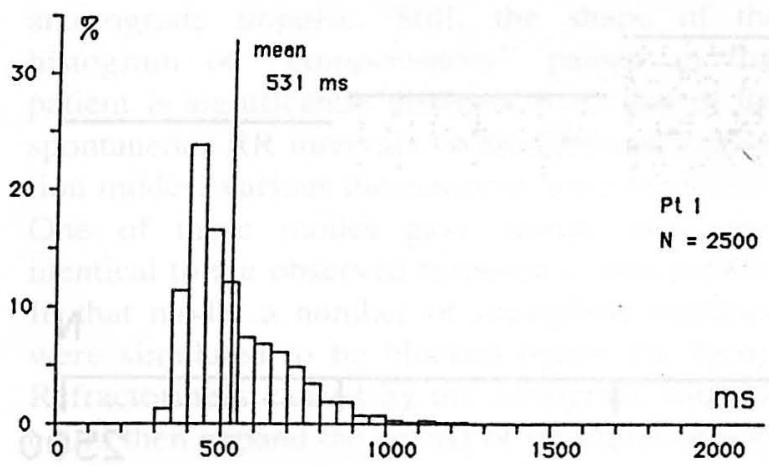

Figure 5. Histogram of simulated spontaneous $R R$ intervals in pt 1. The histogram is insignificantly different from that of the measured spontaneous RR intervals in the same patient. delay determines the degree of interaction between anterograde and retrograde impulses. Individual values of both parameters do not determine the outcome as long as their sum remains constant. Therefore, the simulated optimal retrograde delay may differ from the actual delay in that patient. Furthermore, the retrograde conduction delay in the simulation model also has to cover the latency between the extrastimulus and ventricular activation and the intraventricular conduction delay.

\section{HISTOGRAM OF SIMULATED SR INTERVALS}

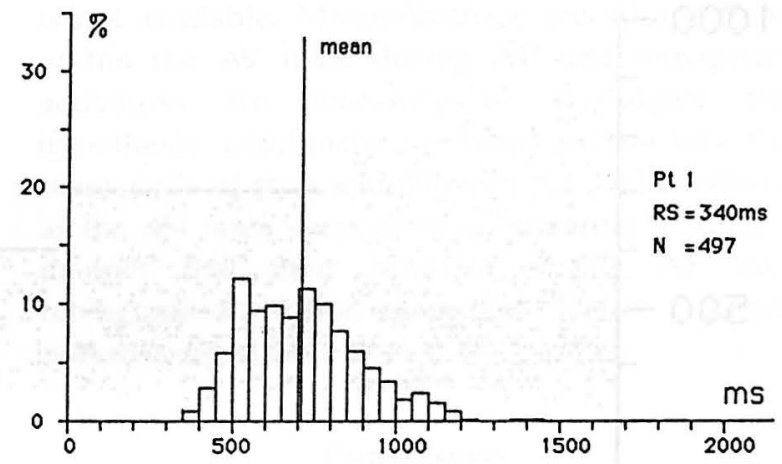

Figure 6. Histogram of simulated SR intervals in pt 1. Anterograde and retrograde conduction delays were set at 100 and $150 \mathrm{~ms}$, respectively. The shape of the histogram is not significantly different from that of the histogram of measured SR intervals as shown in Figure 3. $R S=$ coupling interval between the onset of a spontaneous $R$ wave and the extrastimulus during the simulation; $S R=$ interval between extrastimulus until the onset of the next spontaneous $R$ wave. 
TABLE 3

Results of Simulation

\begin{tabular}{|c|c|c|c|c|c|c|c|}
\hline \multirow[b]{2}{*}{$\begin{array}{l}\text { Patient } \\
\text { Number }\end{array}$} & \multicolumn{2}{|c|}{ Observed SR } & \multicolumn{2}{|c|}{ Retro delay } & \multicolumn{2}{|c|}{ Simulated SR } & \multirow{2}{*}{$\begin{array}{l}\text { Histogram } \\
\text { Shape } \\
\text { Difference }\end{array}$} \\
\hline & $\begin{array}{l}\text { Range } \\
\text { msec }\end{array}$ & $\begin{array}{c}\text { Mean } \\
\text { msec }\end{array}$ & $\begin{array}{c}\text { Range } \\
\text { msec }\end{array}$ & $\begin{array}{l}\text { Optimal* } \\
\text { msec }\end{array}$ & $\begin{array}{c}\text { Range } \\
\text { msec }\end{array}$ & $\begin{array}{c}\text { Mean } \\
\%\end{array}$ & \\
\hline 1 & $420-1010$ & 650 & $100-200$ & 100 & $420-1090$ & 671 & NS \\
\hline 2 & $350-910$ & 578 & $150-200$ & 150 & $390-990$ & 610 & NS \\
\hline 3 & $1040-1810$ & 1407 & $150-300$ & 150 & $1060-1840$ & 1423 & NS \\
\hline 4 & $700-1650$ & 1067 & $100-250$ & 100 & $730-1620$ & 1053 & NS \\
\hline 5 & $460-1940$ & 1065 & $50-300$ & 100 & $490-1970$ & 1034 & NS \\
\hline 6 & $770-1510$ & 1126 & $150-200$ & 150 & $780-1560$ & 1118 & NS \\
\hline 7 & $860-1810$ & 1282 & $100-250$ & 100 & $850-1860$ & 1264 & NS \\
\hline 8 & $840-1740$ & 1241 & - & - & - & - & $\mathrm{S}$ \\
\hline
\end{tabular}

Comparison between observed and simulated "compensatory" pauses. The anterograde conduction delay was set at 100 msec whereas the retrograde delay is varied from 0 to $300 \mathrm{msec}$. The range of retrograde delay values that resulted in a not significant difference between the shapes of observed and simulated SR histograms are listed under "Retro delay." Retro delay = retrograde conduction delay during the simulations. *The "optimal" retrograde delay is defined as the value that resulted in the minimal difference between the mean values of the observed and simulated SR intervals with a not significantly different shape of both histograms. This retrograde delay is used to generate the listed simulated SR intervals. SR = observed compensatory pause following the extrastimulus; $N S=$ not significant; $S=$ significant.

\section{SIMULATED RR INTERVALS}

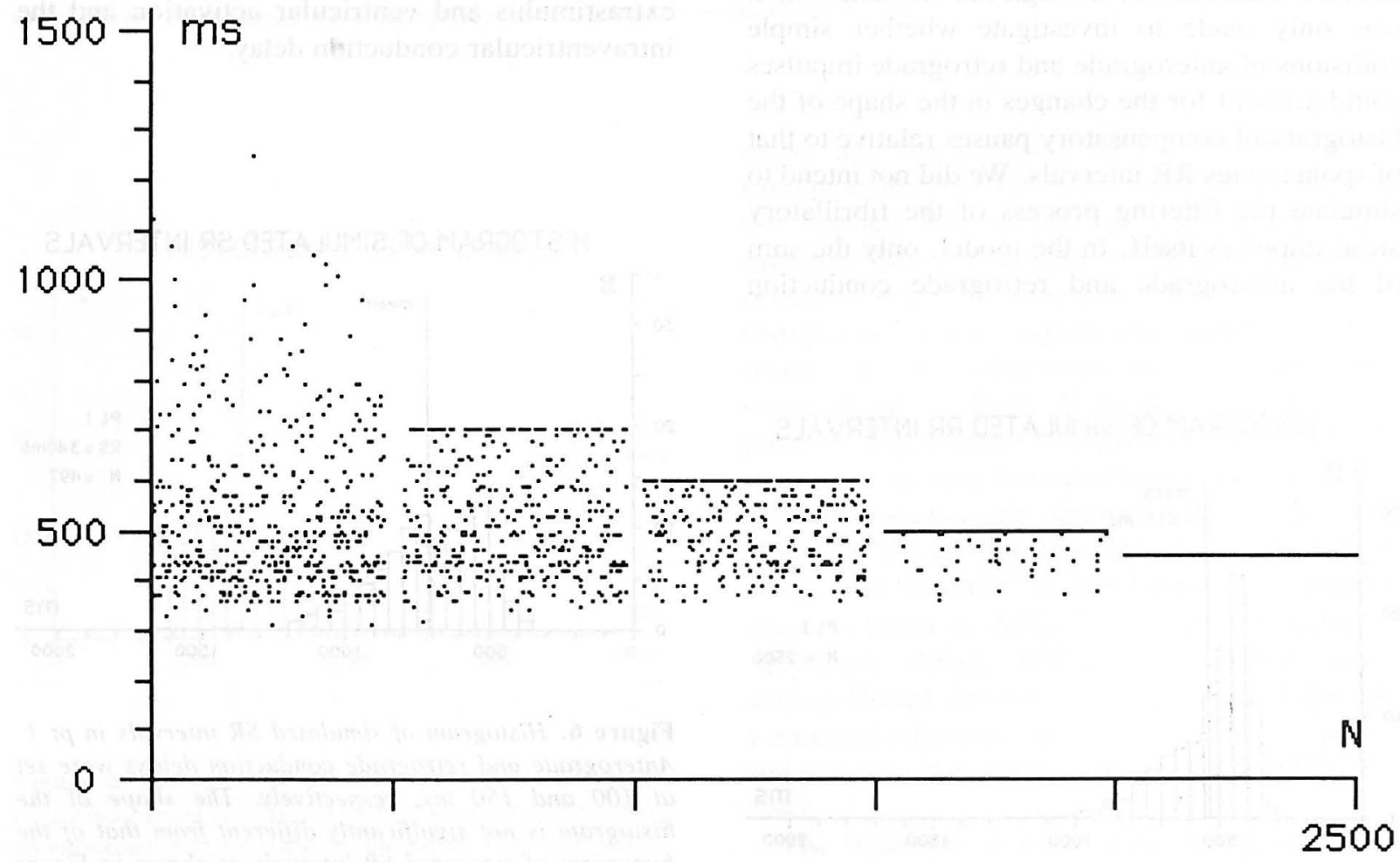

Figure 7. Five episodes of 500 successive simulated RR intervals during a simulation of "on-demand" ventricular stimulation: before pacing and at subsequently decreased pacing intervals of 800, 600, 500, and 450 ms, with 500 RR intervals per step. At the latter pacing interval, the rhythm has become absolutely regular. Compare with Wittkampf et al. (1988), Figure 3. 
Interaction between anterograde and retrograde impulses

In five of the eight patients, the shape of the histograms of SR intervals differed significantly from those of the spontaneous $R R$ intervals. The question rises why in the other three patients this difference was found to be nonsignificant. A plausible explanation for this can be found in the shape of the histogram of their spontaneous RR intervals. The degree of interaction between anterograde and retrograde impulses does not depend only on the relation between the coupling interval of the extrastimulus and the shortest spontaneous RR interval, but more so on the relative number of QRS complexes that would have occurred shortly after the extrastimulus. We therefore calculated the percentage of spontaneous RR intervals that would have occurred within $200 \mathrm{msec}$ after the elicited ventricular extrasystole (Table 2). In four of the five patients in which the shape of the histogram of SR intervals was significantly different from that of the spontaneous RR intervals, this estimated interaction was $66 \%, 77 \%, 53 \%$, and $32 \%$, whereas in the three patients with an insignificant difference between both histograms, this value was $20 \%$ and lower. Thus, for seven of these eight patients with AF and a normally fast ventricular rhythm, this study does not indicate any difference in mechanism of the "compensatory" pause with respect to the patient population of our previous study who have a relatively slow ventricular response (Wittkampf, et al., 1990).

The mechanism in patient 8 remains unclear. From the histogram of spontaneous RR intervals, one would expect that only a small percent of the retrograde impulses would collide with an anterograde impulse. Still, the shape of the histogram of "compensatory" pauses in this patient is significantly different from that of the spontaneous RR intervals. With different simulation modes, various mechanisms were evaluated. One of these modes gave results that were identical to the observed response in this patient. In that mode, a number of retrograde impulses were simulated to be blocked below the focus. Refractoriness caused by the retrograde impulse could then expand the period of interference with anterograde impulses and consequently block anterograde impulses much more frequently than collision of the two wave fronts would otherwise do.

\section{Ventricular on-demand pacing}

The simulated ventricular response during regular ventricular on-demand pacing in the model is strikingly similar to that observed during on-demand pacing in patients with $\mathrm{AF}$ and intact AV conduction (Wittkampf, et al., 1988). The number of short RR intervals decreases with increasing pacing rate until complete stabilization occurs at a pacing interval that is considerably longer than the shortest spontaneous RR interval. Thus the response of the model to this pacing modality closely resembles the clinical response in patients with AF.

\section{Active role of the $A V$ node}

Reset of the timing cycle of AV nodal discharge suggests that retrograde impulses are not blocked by anterograde impulses. This makes concealed conduction at different levels within the node highly unlikely. Rather, these results point to a single hypothetical element within the AV node that behaves as the pacemaker for the ventricles during AF. This does not necessarily imply manifest pacemaker activity, i.e., spontaneous phase 4 depolarization within the AV node (Meijler and Fisch, 1989). Chialvo and Jalife (1987) demonstrated an irregular and chaotic-like response in rhythmically driven nonoscillatory cardiac conducting tissues and reset of distal excitability in a model of discontinuous conduction (Antzelevitch, et al., 1983). Direct experimental evidence of this postulated mechanism is not available. Microelectrode recordings from within the $\mathrm{AV}$ node during $\mathrm{AF}$ and retrograde activation are necessary to investigate the hypothesis. Until then, it remains unclear how the many cells of such a highly-complicated structure as the AV node succeed in cooperating in such a manner that their behavior during $\mathrm{AF}$ and retrograde activation resembles that of a single pacemaking element.

\section{Conclusions}

In patients with chronic AF, the duration of the "compensatory" pause after single premature ventricular complexes may be caused by a combination of two distinctly different mechanisms dependent on the timing of the extrasystole relative to the preceding normal QRS complex. Relatively early retrogradely conducted impulses penetrate into the $\mathrm{AV}$ node and reset its cycle or 
sensitivity to subsequent anterograde impulses. Retrograde conduction of later extrasystoles simply intercepts anterograde impulses below the AV node.

These findings confirm the hypothesis that during $\mathrm{AF}$, the $\mathrm{AV}$ node behaves as the pacemaker for the ventricular rhythm and that the translation of fibrillatory impulses into successful anterograde conduction might be caused by discontinuous electrotonically-mediated propagation within the AV node.

Acknowledgments: We express our gratitude for the encouragement and constructive comments of Etienne O. Robles de Medina, M.D., University Hospital of Utrecht. We thank Jan Res, M.D., Asterdam; Wim L. Mosterd, M.D., and Henk van Rooyen, Amersfoort, who selected the patients and assisted during the measurements. Part of the equipment was kindly supplied by Vitatron Medical BV, Dieren, The Netherlands.

\section{References}

Antzelevitch C, Moe G: Electrotonic inhibition and summation of impulse conduction in mammalian Purkinje fibers. Am J Physiol (Heart Circ Physiol) 1983; 245:H42- H53.

Billette J: Atrioventricular nodal activation during premature stimulation of the atrium. Am J Physiol (Heart Circ Physiol 21) 1987;252:163-177.

Bootsma BK, Hoelen AJ, Strackee J, Meijler FL: Analysis of R-R intervals in patients with atrial fibrillation at rest and during exercise. Circulation 1970;41:783794.

Brody DA: Ventricular rate patterns in atrial fibrillation. Circulation 1970;41:733-735.

Chialvo DR, Jalife J: Non-linear dynamics of cardiac excitation and impulse propagation. Nature 1987;330: 749-752.
Dreifus LS, Mazgalev T: “Atrial paralysis:” Does it explain the irregular ventricular rate during atrial fibrillation? J Am Coll Cardiol 1988;11:546-547.

Jalife J: The sucrose gap preparation as a model of AV nodal transmission: Are dual pathways necessary for reciprocation and AV nodal "echoes"? PACE 1983:6: 1106-1122.

Langendorf R: Concealed AV conduction: The effect of blocked impulses on the formation and conduction of subsequent impulses. Am Heart J 1948;35:542-552.

Langendorf R, Pick A, Katz LN: Ventricular response in atrial fibrillation: Role of concealed conduction in the AV node. Circulation 1965;32:69-75.

Lehmann MH, Mahmud R, Denker S, Soni J, et al: Retrograde concealed conduction in the atrioventricular node: Differential manifestations related to level of intranodal penetration. Circulation 1984;70:392-401.

McKinnie J, Avitall B, Caceres J, Jazayeri M, et al: Electrophysiologic spectrum of concealed intranodal conduction during atrial rate acceleration in a model of 2:1 atrioventricular block. Circulation 1989;80:43-50.

Meijler FL, Fisch C: Does the atrioventricular node conduct? Br Heart J 1989;61:309-315.

Moe GK, Abildskov JA, Mendez C: An experimental study of concealed conduction. Am Heart J 1964;67:338356.

Moore EN: Observations on concealed conduction in atrial fibrillation. Circ Res 1967;21:201-208.

Rosenbleuth A: Functional refractory penod of cardiac tissues. Am J Physiol 1958;194:171-183.

Wittkampf FHM, de Jongste MJL, Lie KI, Meijler FL: Effect of right ventricular pacing on ventricular rhythm during atrial fibrillation. J Am Coll Cardiol 1988; 11:539-545.

Wittkampf FHM, de Jongste MJL, Meijler FL: Atrioventricular nodal response to retrograde activation in atrial fibrillation. J Cardiovasc Electrophysiol 1990; 1:437-447.

Zar JH: The Kolmogorov-Smirnov goodness of fit. In: McElroy WD, Swanson CP, eds. Biostatistical Analysis. Prentice-Hall, Inc., Englewood Cliffs, 1974; p.54. 